\title{
Bread Crumbs: A Technique for Modelling Large Outdoor Ground Features
}

\author{
Wayne Piekarski and Bruce H. Thomas \\ Wearable Computer Laboratory \\ School of Computer and Information Science \\ University of South Australia \\ Mawson Lakes, SA, 5095, Australia \\ \{wayne, thomas\}@cs.unisa.edu.au
}

\begin{abstract}
This paper presents a new technique we have created, known as Bread Crumbs, which allows the modelling of large outdoor ground features using a mobile augmented reality system and a user's physical presence. Using this technique, we demonstrate an example of modelling a large grassy area on campus. This technique is one component of a larger set of tools which allow users to capture and work with complex outdoor geometry interactively, and to view this geometry in new and interesting ways.
\end{abstract}

\section{Introduction}

This paper presents the new Bread Crumbs technique we have developed that allows users to capture large outdoor ground features using a mobile augmented reality (AR) system. Traditionally, AR systems have always required 3D models from other sources, and this process can be error prone and time consuming, requiring detailed information which may not be easily available.

The Bread Crumbs technique, as well as the techniques described previously in [4], are designed to allow the capture of outdoor geometry with a mobile AR system and no prior information. By leveraging information about the user's physical presence in the world and using an AR display, we can enter data and immediately verify the accuracy of it in real time. With these new techniques, an autonomous user can capture 3D geometry any time, any place, and anywhere on Earth, with user interactions that are based on hand and head gestures. While it is possible to capture some outdoor objects using multiple cameras or laser scanners, these techniques have a number of limitations such as requiring line of sight to the object, which might not be possible. The techniques we have developed have their own limitations [4], and so complement existing techniques rather than replacing them - and also provide a useful scenario for AR user interface research.

\section{Current Tinmith-Metro modeller}

We have developed a modelling application known as Tinmith-Metro [4] which allows users to walk around in an outdoor environment, capturing the geometry of existing ob-

For more information, as well as videos of the system in use outdoors, readers are invited to visit our web site at http://www.tinmith.net jects, and also creating new objects. The user interface for this application is quite complex and has a large number of commands, and making these all accessible in an intuitive fashion is important. Since traditional desktop interaction devices such as keyboards and mice are not suitable for outdoor mobile computing, new techniques for controlling the system were developed. The primary input device is a set of custom built gloves with metallic pads, allowing the system to detect finger to thumb and finger to palm pinch gestures. Figure 1 shows an example of the system being controlled by these gloves in an outdoor setting. At the bottom left and right are the menu strips, indicating the options that will be executed if the matching finger is pressed. The user navigates through the menu to perform modelling operations using a series of pinch and palm gestures. Using fiducial markers on the glove, cameras track the location of the hands in $3 \mathrm{D}$, and cursors are superimposed to indicate the tracking of the hands. By combining finger pinches with tracking, the set of gloves is the only user input device required by the system.

Previously in [4] we demonstrated an example application which allowed users to capture the geometry of simple building models using line of sight techniques (infinite planes), and to place down prefabricated objects at real world locations (street furniture). In many cases, graphical objects that model physical objects can be instantiated from a template, which is easier to reuse rather than create new ones from scratch. Furthermore, some small objects contain intricate details that may exceed the resolution of the tracking devices.

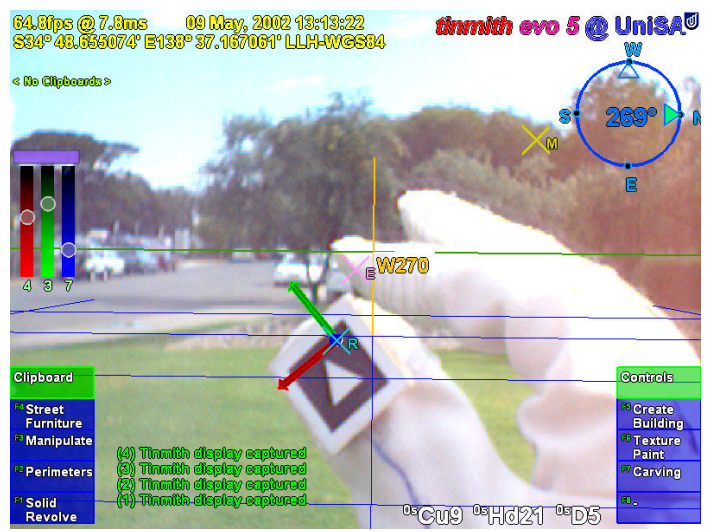

Figure 1 - Tinmith immersive augmented reality display, showing vertical menus, on screen gadgets, gloves, and 3D/2D overlaid tracking cursors 


\section{Bread crumbs}

Some complex outdoor shapes such as rivers, lakes, and long twisting roads are inefficient to model using the infinite planes technique because it is designed to model approximately convex objects. In many cases it is possible to walk near or on top of the edges of these large natural features, and so a more direct system of marking vertices is possible. The Bread Crumbs technique is inspired by the children's fairytale Hansel and Gretel [1]. In this story, the children are taken out into the forest by their parents in the hope they will not come back home again. However, Hansel was carrying a loaf of bread and dropped small crumbs of bread where they walked, enabling them to find their way back home again. On another occasion, the children found themselves unable to get home because the birds in the forest ate up the trail.

With a mobile augmented reality system, it is possible to place down less edible and more reliable virtual markers onto the ground to simulate bread crumbs. The user walks along the perimeter of the object they wish to model, and places markers at points of interest (see Figure 2) using the same interactions as the street furniture technique. When the user fully walks around the object, a closed perimeter is formed. Initially the perimeter defined is a thin polygon, but can be extruded up to define a solid building outline, or extruded down to approximate the bottom of a lake or river. The resulting object can then be operated on using other techniques to add other features to the geometry. While it may seem that this technique offers no more than using a handheld GPS unit, it should be noted that this technique has a number of new ideas - the verification of marker placement in real time with AR; viewing overall object layout from external VR style views; adjusting markers using simple hand gestures; the ability to create solid shapes out of these perimeters to use them as input objects to form even more complex geometry.

We have used the Bread Crumbs technique to model roads, parking lots, some low buildings, grassy areas on our campus (see Figure 2 and Figure 3), and other concave outline style shapes. It is also possible to lay down trails similar to the original fairytale, indicating paths and steering directions to navigate in the physical world while using the system.

\section{External views}

HMDs are mainly used to overlay virtual objects onto the user's view of the world in real time. For our modelling techniques, AR allows the user to verify the resulting $3 \mathrm{D}$ models. However, in some cases it is more useful to work in an external VR style view (such as orbital view [2]) with the live video overlay replaced with a sky and ground texture on the HMD. In the Bread Crumbs technique, we found that it is more intuitive to use the technique while working in an external VR view (see Figure 2) than in the immersive AR view with a limited field of view (see Figure 3), as the user has a higher situational awareness of the objects that have been placed around them. The geometry of many shapes only becomes obvious when viewed from a birds eye aerial position

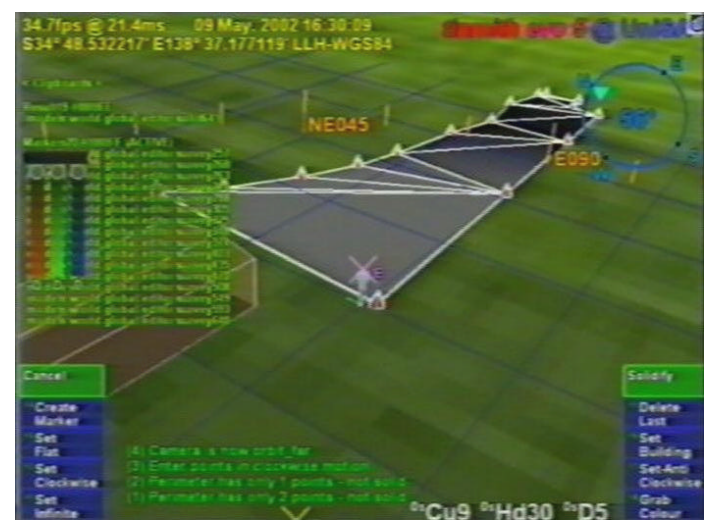

Figure 2 - VR view of marker cones defining a flat concave perimeter

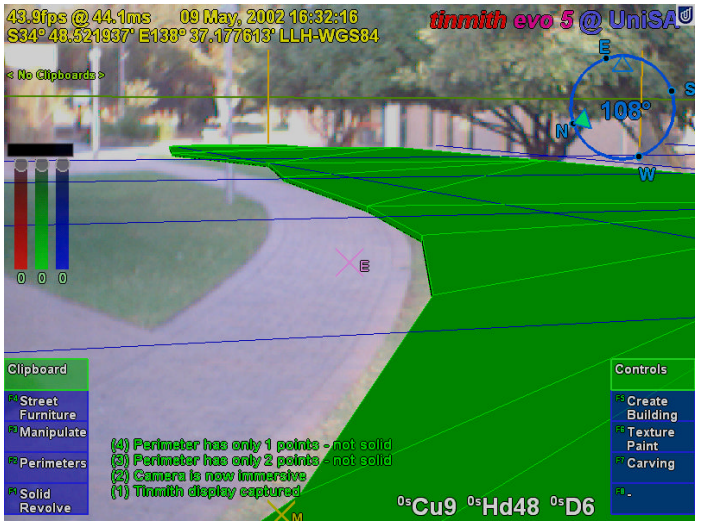

Figure 3-AR view showing registration of perimeter to physical world

instead of at ground level. By interfacing an indoor VR system running in orbital mode with the outdoor AR system, it is possible for users indoors to observe what objects are being created outdoors [3].

\section{Conclusion}

This paper has presented the Bread Crumbs technique, which is designed to use a user's physical presence, as well as mobile AR systems, to capture the geometry of large outdoor features. We demonstrated this technique with an example, presented using both traditional immersive AR and also external orbital views.

\section{References}

[1] Grimm, J. and Grimm, W. Hansel and Gretel - Kinder- und Hausmärchen. Germany, 1822.

[2] Koller, D. R., Mine, M. R., and Hudson, S. E. Head-Tracked Orbital Viewing: An Interaction Technique for Immersive Virtual Environments. In 9th Int'l Symposium on User Interface Software Technology, pp 81-82, Seattle, Wa, Nov 1996.

[3] Piekarski, W., Gunther, B., and Thomas, B. Integrating Virtual and Augmented Realities in an Outdoor Application. In 2nd Int'l Workshop on Augmented Reality, pp 45-54, San Francisco, Ca, Oct 1999.

[4] Piekarski, W. and Thomas, B. Tinmith-Metro: New Outdoor Techniques for Creating City Models with an Augmented Reality Wearable Computer. In 5th Int'l Symposium on Wearable Computers, pp 31-38, Zurich, Switzerland, Oct 2001. 